\title{
Fournier Gangrene in a Patient With Type 2 Diabetes Mellitus Treated With Dapagliflozin: A Case Report
}

\author{
Jae Young Moon, Min Ro Lee, Jong Hun Kim, Gi Won Ha \\ Research Institute of Clinical Medicine of Jeonbuk National University-Biomedical Research Institute of Jeonbuk National University Hospital, \\ Jeonju, Korea
}

Rare cases of Fournier gangrene (FG) possibly associated with sodium-glucose cotransporter 2 inhibitors have been reported. We present a case of a 66-year-old male patient with type 2 diabetes mellitus on oral metformin, glimepiride, and dapagliflozin therapy. He presented with pain in the perineum and scrotum for 5 days. The clinical finding, computed tomography finding, and laboratory data were matched with FG. Emergency surgical drainage, debridement of necrotic tissue, and diverting loop ileostomy formation were performed by a urologist and a surgeon. The patient had no complications from diabetes before the onset of FG, and serum glucose management was good at the onset of FG. This case shows an FG patient with good glucose management taking dapagliflozin and suggests a possible association between dapagliflozin and FG. Further evaluation and additional research on this relationship are needed.

\section{Keywords: Fournier gangrene; Dapagliflozin; Type 2 diabetes mellitus}

\section{INTRODUCTION}

Fournier gangrene (FG), first described by Jean Alfred Fournier in 1883 [1], is an adverse polymicrobial infection that typically results in cell death of the perineal and genital fasciae. Further, FG needs early recognition, aggressive debridement of devitalized tissue, antibiotics therapy, and a search for primary sources as the treatment of choice for FG [2]. As defined, FG is considered to be idiopathic. Various risk factors for FG have been reported, including diabetes mellitus (DM), alcohol abuse, poor hygiene, malignancies, and human immunodeficiency virus infections. Most of these risk factors have similar features, including impairment and immunosuppression in the microcirculation [3].

Glycemic control is improved by sodium-glucose cotransporter 2 (SGLT2) inhibitors through inhibiting glucose reabsorption in

Received: Feb 12, 2020 - Revised: Jun 11, 2020 - Accepted: Jun 22, 2020 Correspondence to: Gi Won Ha, M.D.

Research Institute of Clinical Medicine of Jeonbuk National UniversityBiomedical Research Institute of Jeonbuk National University Hospital, 20 Geonji-ro, Deokjin-gu, Jeonju 54907, Korea

Tel: +82-63-250-1570, Fax: +82-63-271-6197

E-mail: acts29@jbnu.ac.kr

ORCID: https://orcid.org/0000-0001-9401-1760

(C) 2021 The Korean Society of Coloproctology

This is an open-access article distributed under the terms of the Creative Commons Attribution NonCommercial License (https://creativecommons.org/licenses/by-nc/4.0) which permits unrestricted noncommercial use, distribution, and reproduction in any medium, provided the original work is properly cited. kidney tubules, which thereby increases glucose excretion through urine [4]. To date, the U.S. Food and Drug Administration (FDA) has approved 4 SGLT2 inhibitors: dapagliflozin, empagliflozin, ertugliflozin, and canagliflozin [5]. SGLT2 inhibitors usage has been correlated with a higher risk of genital infection following increased glycosuria [6]. This has prompted the FDA to publish a warning about FG occurring in patients undergoing SGLT2 inhibitor therapy [7]. We describe the first patient who developed FG during dapagliflozin treatment at our institution.

\section{CASE REPORT}

This study was approved by the Institutional Review Board of Jeonbuk National University Hospital (2020-06-012). The written informed consent and permission to use clinical images were received from the patient.

A 66-year-old male patient came to our hospital with a 5-day history of pain in the scrotal and perianal area. The patient's history revealed type 2 DM (T2DM), hypertension, acute myocardial infarction (percutaneous coronary intervention done 2 times in 2007 and 2014), smoking, and alcohol intake. He has been taking metformin 1,000 mg/day, glimepiride $4 \mathrm{mg} /$ day, and dapagliflozin $10 \mathrm{mg} /$ day as antidiabetic mediation. He started taking dapagliflozin in March 2017. He had no complications from DM and had well-controlled hygiene.

Visiting our hospital, his body temperature was $36.3^{\circ} \mathrm{C}$. He was 

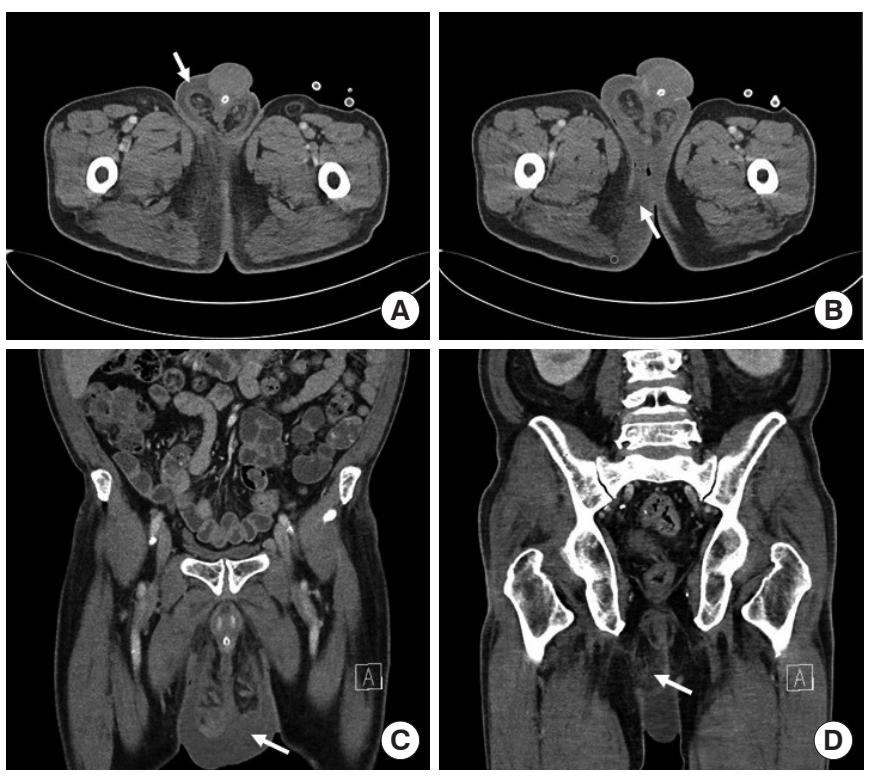

Fig. 1. Computed tomography scans of the lower abdomen (A, B) and pelvis (C, D) showing pathologic Fournier gangrene (arrows).

not in a state of shock, and vital signs were stable: blood pressure, 99/60 mmHg; heart rate, 105 beats per minute; and respiratory rate, 20 breaths per minute. Skin redness, induration, swelling, and tenderness were observed in both the scrotum and perineum. Focal skin necrosis and a foul smell were observed, especially in the lower scrotal area. There was no trauma.

Routine laboratory data revealed a white blood cell (WBC) count of $15.03 \times 10^{3} / \mu \mathrm{L}, \mathrm{C}$-reactive protein at $211.46 \mathrm{mg} / \mathrm{L}$, and hemoglobin A1c (HbAlc) at 6.7\%. Urinary analysis showed no evidence of urinary infection (red blood cells, 0 to 2/high power field [HPF]; WBC, 3-5/HPF). A computed tomography (CT) scan of the lower abdomen and pelvis revealed findings consistent with FG (Fig. 1).

All antidiabetic drugs were stopped at admission. In the emergency room, a Foley catheter was inserted, and antibiotics ( $2 \mathrm{~g}$ of ceftriaxone and $500 \mathrm{mg}$ of metronidazole) were given intravenously. The patient underwent emergency surgery under general anesthesia, and laparoscopy-assisted diverting loop ileostomy formation, surgical drainage, and debridement of the necrotic tissue on scrotum, groin, and perineum were performed. Due to the perioperative onset of shock with a need for catecholamine (0.05$\mu \mathrm{g} /$ minute of norepinephrine) during the operation, the patient was admitted to the surgical intensive care unit (SICU). In the SICU, we changed antibiotics to more broad-spectrum antibiotics ( $4 \mathrm{~g}$ of piperacillin with $0.5 \mathrm{~g}$ of tazobactam). On postoperative day (POD) 1 , the patient's vital signs stabilized and he was transferred to a ward. On POD 2, a diet was started and antidiabetic drugs resumed without dapagliflozin. On POD 6, $100 \mathrm{mg}$ of sitagliptin was added after an endocrinology consultation. On POD 7 , wound debridement was performed under local anesthesia

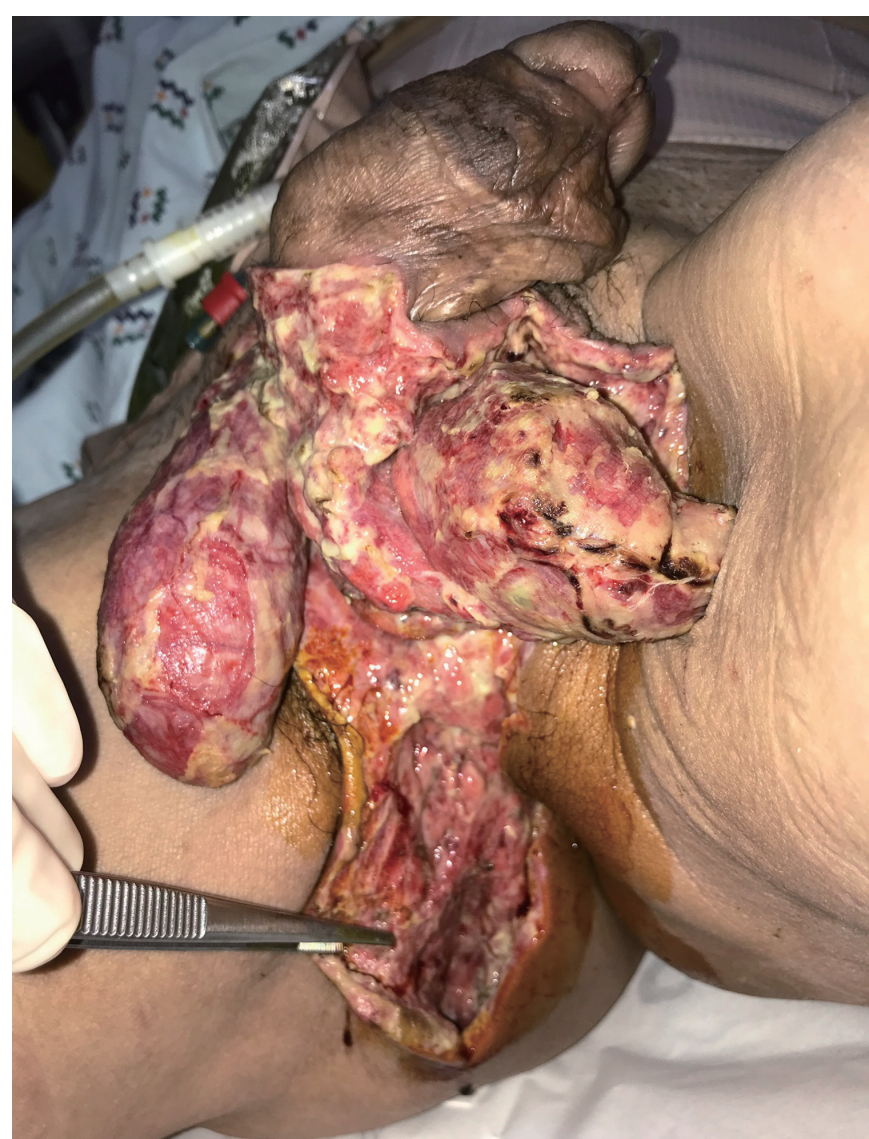

Fig. 2. An image of the groin and scrotum on postoperative day 8 .

(Fig. 2). The patient was discharged on POD 15 and transferred to a local hospital for further dressing of the surgical wound with this antidiabetic medication.

\section{DISCUSSION}

DM usually presents with significant microvascular manifestations, as suggested by diabetic retinopathy, amputation, and nephropathy. FG may result from similar processes and may worsen as a result of relative immunosuppression. During the infection period, endogenous production of glucose increases due to an increased rate of catabolism. Adherence, chemotaxis, and bactericidal activities of phagocytes are affected by hyperglycemia; and reports show its detrimental effects on cellular immunity. Further, DM has been reported to be a predisposing factor in $32 \%$ to $66 \%$ of FG cases. Moreover, DM impacts the clinical course of the disease, associated with a longer hospital stay, younger age, and a different bacteriology spectrum [8].

One of the common causes of FG is anal fistula. According to the patient's past history, physical exam, and CT review, we cannot determine suspicion for anal fistula. And in the operation field, we couldn't find internal opening of anal fistula. So it's hard 
to conclude that this case is related to anal fistula.

Among patients receiving SGLT2 inhibitors between March 2013 and January 2019, the FDA identified 55 cases of FG. Comparatively, only 19 FG cases were reported between 1984 and January 2019 among patients receiving different antidiabetic agents [7]. If FG were correlated with only DM and not SGLT2 inhibitors, significantly more cases should have been reported with different antidiabetic agents, especially in consideration of the larger 35-year timeframe and a greater number of agents. Nevertheless, there have been too few cases to have confidence that FG is related to SGLT2 inhibitor usage.

There are some case reports on FG in patients undergoing SGLT 2 therapy. Kumar et al. [9] reported a 41-year-old male patient with T2DM with an HbA1c of $11.2 \%$ using empagliflozin, and Onder et al. [10] reported a 64-year-old male patient with T2DM with an $\mathrm{HbAlc}$ of $7.4 \%$ using dapagliflozin. However, similar to our case, Nagano et al. [11] reported a 34-year-old male patient with T2DM with an HbA1c of 6.5\% at the time of FG onset who was on sitagliptin, glibenclamide, and empagliflozin therapy. The patient had no additional complications from DM besides FG [11]. Thus, the risk for developing FG remains in spite of good glycemic management with SGLT2 inhibitors.

Glycosuria can be anticipated with SGLT2 inhibitor treatment and is correlated with increased risk of urinary tract and urogenital infections [12, 13]. Additionally, the environment providing glycosuria-enhanced growth of urogenital flora also may provide an ideal environment for FG as the perineum is colonized already with organisms from the gastrointestinal tract [7].

Additional SGLT2 therapy and deranged glucose levels in the blood may lead potentially to extreme glucose levels in urine, which can provide a favorable environment for urinary infection. Coupled with local immunodeficiency (caused by DM and other comorbidities) and microvascular supply impairment, FG may result in some patients [14]. Nevertheless, the causal mechanism underlying the relationship between SGLT2 inhibitors and FG is still not understood. So FG among patients receiving SGLT 2 inhibitors is not always related to urinary tract infection.

In conclusion, FG only occurs in a minimum number of patients receiving SGLT 2 inhibitors. But a possible association between FG and SGLT2 inhibitors may exist, and patients treated with SGLT 2 inhibitors should be warned about the risk of FG, controlling hygiene, smoking, and quitting alcohol. Carefully monitored for its symptoms at follow up. And if FG occurs in those patients with SGLT 2 inhibitors, patients should visit the hospital immediately for proper management, and SGLT 2 inhibitors should be discontinued.

\section{CONFLICT OF INTEREST}

No potential conflict of interest relevant to this article was reported.

\section{REFERENCES}

1. Fournier JA. Jean-Alfred Fournier 1832-1914. Gangrène foudroyante de la verge (overwhelming gangrene). Sem Med 1883. Dis Colon Rectum 1988;31:984-8.

2. Park JH, Park SC, Jeon HM, Jung JH, Kim WW, Oh ST, et al. Clinical analysis of Fournier's gangrene. J Korean Soc Coloproctol 2000;16:309-15.

3. Chennamsetty A, Khourdaji I, Burks F, Killinger KA. Contemporary diagnosis and management of Fournier's gangrene. Ther Adv Urol 2015;7:203-15.

4. Riser Taylor S, Harris KB. The clinical efficacy and safety of sodium glucose cotransporter-2 inhibitors in adults with type 2 diabetes mellitus. Pharmacotherapy 2013;33:984-99.

5. Powell J, Garland SG. Ertugliflozin: a new option in the SGLT-2 inhibitor market for the treatment of type 2 diabetes mellitus. Ann Pharmacother 2019;53:478-85.

6. Cefalu WT, Riddle MC. SGLT2 inhibitors: the latest "new kids on the block"! Diabetes Care 2015;38:352-4.

7. Bersoff-Matcha SJ, Chamberlain C, Cao C, Kortepeter C, Chong $\mathrm{WH}$. Fournier gangrene associated with sodium-glucose cotransporter-2 inhibitors: a review of spontaneous postmarketing cases. Ann Intern Med 2019;170:764-9.

8. Nisbet AA, Thompson IM. Impact of diabetes mellitus on the presentation and outcomes of Fournier's gangrene. Urology 2002; 60:775-9.

9. Kumar S, Costello AJ, Colman PG. Fournier's gangrene in a man on empagliflozin for treatment of type 2 diabetes. Diabet Med 2017;34:1646-8.

10. Onder CE, Gursoy K, Kuskonmaz SM, Kocer U, Culha C. Fournier's gangrene in a patient on dapagliflozin treatment for type 2 diabetes. J Diabetes 2019;11:348-50.

11. Nagano Y, Yakame NK, Aoki H, Yamakawa T, Kondo NI. Fournier's gangrene in a patient with type 2 diabetes mellitus treated with empagliflozin: a case report. Drug Saf Case Rep 2019;6:11.

12. Sarnoski-Brocavich S, Hilas O. Canagliflozin (invokana), a novel oral agent for type-2 diabetes. P T 2013;38:656-66.

13. Johnsson KM, Ptaszynska A, Schmitz B, Sugg J, Parikh SJ, List JF. Vulvovaginitis and balanitis in patients with diabetes treated with dapagliflozin. J Diabetes Complications 2013;27:479-84.

14. Rodler S, Weig T, Finkenzeller C, Stief C, Staehler M. Fournier's gangrene under sodium-glucose cotransporter 2 inhibitor therapy as a life-threatening adverse event: a case report and review of the literature. Cureus 2019;11:e5778. 